\title{
Impact of "increased-risk" donor hearts on transplant outcomes: A propensity-matched analysis
}

Yasuhiro Shudo, MD, PhD, Jeffrey E. Cohen, MD, Bharathi Lingala, PhD, Hao He, PhD, Yuanjia Zhu, MD, and Y. Joseph Woo, MD

\section{ABSTRACT}

Objectives: Orthotopic heart transplantation (OHT) remains the gold standard for advanced heart failure. Increased risk (IR) donors were categorized by the United Network for Organ Sharing Database (UNOS) according to the Centers for Disease Control and Prevention (CDC) criteria. However, the impact of CDC IR donor hearts on the outcome of adult OHT recipients remains unclear. The aim of this study was to compare the outcome of adult OHT recipients between CDC IR and non-CDC IR donor grafts.

Methods: Data were obtained from the United Network for Organ Sharing Databas. All adult patients (age $\geq 18$ years) undergoing OHT from 2004 through 2016 were included $(n=24,751)$. Propensity scores for CDC IR donors were calculated by estimating probabilities of CDC IR donor graft use using a nonparsimonious multivariable logistic regression model. Patients were matched 1:1 using a greedy matching algorithm based on the propensity score of each patient. The impact of CDC IR donors on the post-transplant outcomes, such as 30-day and overall mortalities, was investigated using Cox-proportional hazards. Overall survival probability analyses were performed.

Results: Of 24,751 primary heart transplants from 2004 to 2016 with 3584 $(14.5 \%)$ as IR donors, 6304 transplants were successfully matched $(n=3152$ in CDC IR group and non-IR group). There were no significant differences in baseline characteristics in recipients and donors. In the Cox-proportional hazards model for matched subjects, the use of CDC IR grafts was not associated with 30-day (hazard ratio of IR group vs non-IR group $0.97 ; 95 \%$ confidence interval, $0.87-1.08 ; P=.57$ ) and overall mortalities (hazard ratio, $0.94 ; 95 \%$ confidence interval, $0.73-1.21 ; P=.62$ ). Interestingly, post-transplant acute myocardial rejection episodes during hospital stays were found more often in the CDC-IR group, compared with the non-CDC IR group (CDC IR, $\mathrm{n}=358$ [11.4\%]; non-CDC IR, $\mathrm{n}=304[9.6 \%] P=.03$ ), whereas post-transplant pacemaker placements were performed less frequently in the CDC IR group (CDC IR, $\mathrm{n}=80[2.6 \%]$; non-CDC IR, $\mathrm{n}=111[3.5 \%] P=.020)$. Importantly, there was no significant difference in the overall survival probability between CDC IR and non-IR groups in both unadjusted and adjusted survival analyses.

Conclusions: CDC IR status does not have a significant impact on adult OHT recipient survival probability. Increased use of CDC IR donor grafts can potentially alleviate the persistent and worsening shortage of available donor organs and shorten the waitlist time for heart transplantation. (J Thorac Cardiovasc Surg 2019;157:603-10)

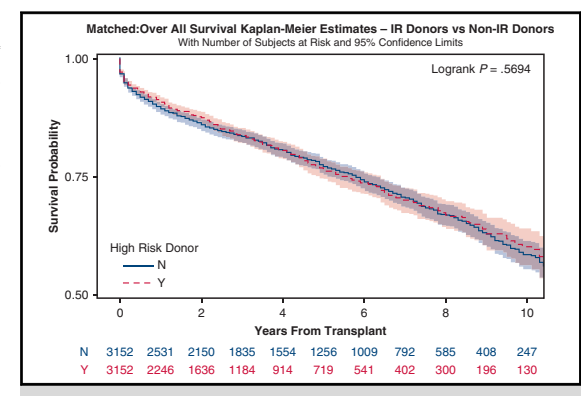

$C D C$, Centers for Disease Control and Prevention.

\section{Central Message}

The equivalent survival reported at long-term follow-up in adult orthotopic heart transplantation recipients is encouraging in the use of increased-risk donor grafts.

\section{Perspective}

CDC increased-risk status does not have a significant impact on adult orthotopic heart transplantation recipient survival probability. Increased use of CDC increased risk donor grafts can potentially improve the persistent and worsening shortage of available donor organs and shorten the waitlist times for heart transplantation.

\section{See Editorial page 611.}

See Editorial Commentary page 613.

\footnotetext{
From the Department of Cardiothoracic Surgery, Stanford University School of Medicine, Stanford, Calif.

Read at the 98th Annual Meeting of The American Association for Thoracic Surgery, San Diego, California, April 28-May 1, 2018.

Received for publication Jan 15, 2018; revisions received June 8, 2018; accepted for publication Aug 7, 2018.
}

\footnotetext{
Address for reprints: Y. Joseph Woo, MD, Falk Cardiovascular Research Center, Stanford University School of Medicine, 300 Pasteur Drive, Stanford, CA 94305 (E-mail: joswoo@stanford.edu).

$0022-5223 / \$ 36.00$

Copyright (C) 2018 by The American Association for Thoracic Surgery

https://doi.org/10.1016/j.jtcvs.2018.08.120
} 


\section{Abbreviations and Acronyms \\ $\mathrm{CDC}=$ Centers for Disease Control and Prevention \\ $\mathrm{ECMO}=$ extra-corporeal membrane oxygenation \\ $\mathrm{HCV}=$ hepatitis $\mathrm{C}$ virus \\ HIV = human immunodeficiency virus \\ IABP $=$ intra-aortic balloon pump \\ IR $=$ increased risk \\ NAT $=$ nucleic acid testing \\ OHT $=$ orthotopic heart transplantation \\ UNOS = United Network for Organ Sharing \\ Database}

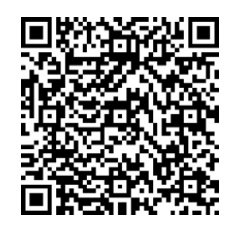

Heart disease is the leading cause of death in the United States. Advanced or medically refractory heart failure represents the end-stage form of heart disease. ${ }^{1}$ Many treatment options have been developed for patients with end-stage heart failure, among which orthotopic heart transplantation (OHT) remains the gold standard. ${ }^{2}$ However, the persistent shortage of available donor organs has resulted in an ever-increasing waitlist for transplantation, as well as longer waiting periods before surgery. Because of the persistent and worsening shortage of available donor hearts, we have previously proposed alternative approaches to maximize organ allocation including using marginally acceptable organs, ${ }^{3}$ harvesting donor hearts from more distant locations, accepting longer cold ischemic times, and applying a domino heart transplantation strategy. ${ }^{4}$

High-risk behavior donors for infection were categorized by the United Network for Organ Sharing Database (UNOS) according to the Centers for Disease Control and Prevention (CDC) criteria in 2004. Since 2013, the high-risk behavior donor category was replaced by an increased-risk (IR) behavior donor category for infection. To summarize, a donor will be categorized as a CDC IR if the donor meets any of the following criteria: (1) men who have had sex with men; (2) individuals who report nonmedical injection of drugs; (3) individuals with hemophilia or related clotting disorders who have received human-derived clotting factor concentrates; (4) individuals who have engaged in sex with any individual described previously or with an individual known or suspected to have human immunodeficiency virus (HIV) infection; (5) individuals who have been exposed to HIV-infected blood; and (6) inmates of correctional systems. Organ-procurement organizations are required to notify transplant surgeons when a donor meets these CDC IR criteria, and an additional informed consent process is required. A significant number of medically suitable organs from IR donors are not transplanted. ${ }^{5}$ Nevertheless, the impact of CDC IR donor hearts on adult OHT recipients' outcomes remains unclear. Therefore, we seek to evaluate the impact of CDC IR donor grafts by comparing outcomes of adult OHT recipients using CDC IR versus non-CDC IR donor grafts.

\section{METHODS \\ Patient Selection}

The UNOS registry was analyzed for all patients equal to and older than 18 years old who underwent OHT (Video 1) between January 1, 2004, and December 31, 2016. Patients were excluded if they were 18 years or younger, did not undergo isolated heart transplantation, or underwent reheart transplantation. Patients with incomplete data were excluded from the analysis. Then, the patient data were divided into 2 groups based on donor CDC risk status according to the CDC IR donor criteria in 2013.

The primary endpoint was graft survival, with graft loss being defined as patient death or re-heart transplantation. The secondary endpoint was transplant-related morbidity, such as acute rejection episodes or permanent pacemaker placement during the hospital stay. Studies using this data set have been determined to be exempt from review by the Institutional Review Board of Stanford University School of Medicine.

\section{Propensity Score Matching}

Propensity scores for CDC IR donor graft use were calculated using a nonparsimonious multivariable logistic regression model to include recipient baseline characteristics (age, sex, body mass index, blood type), medical history (diabetes, renal function, and liver function), etiology of heart failure, total waiting time, and preoperative life support (hospitalization in intensive care unit, intra-aortic balloon pump [IABP], extracorporeal membrane oxygenation [ECMO], durable ventricular-assist device). Donor characteristics used in the model included baseline characteristics (age, sex, body mass index, blood type), medical history (diabetes, hypertension, cocaine usage, hepatitis $\mathrm{C}$ status, coronary artery disease), left ventricular ejection fraction, and allograft ischemic time. CDC IR and non-IR patients were matched 1:1 using a greedy matching algorithm (nearest match without replacement) based on the propensity score of each patient. A caliper width of 0.2 of the standard deviation of the logit of the propensity

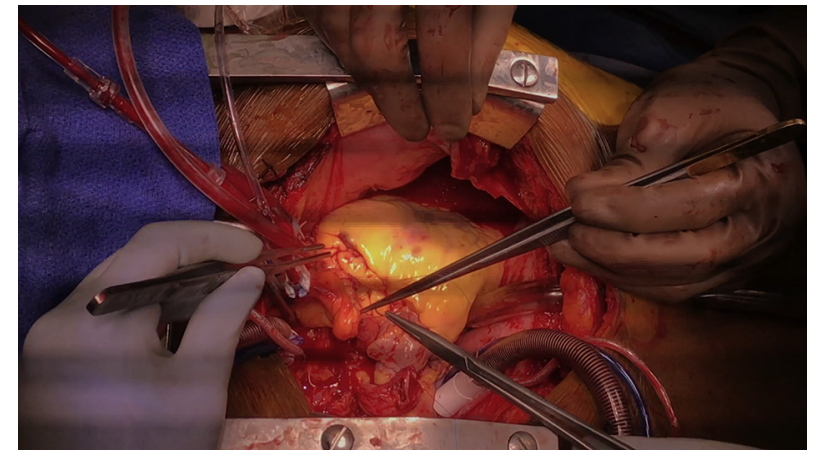

VIDEO 1. Operative video of orthotopic heart transplantation (OHT). Video available at: https://www.jtcvs.org/article/S0022-5223(18)32816-2/ fulltext. 
score was applied as the matching criteria. ${ }^{6}$ An absolute standardized mean difference of $<0.02$ was considered to represent relative balance. ${ }^{7}$ Only patients who were successfully matched were used in the assessment of CDC IR graft survival.

\section{Statistical Analysis}

Continuous variables were analyzed using a 2-sample $t$ test, and categorical variables were analyzed using $\chi^{2}$ test. After propensity matching, the impact of CDC IR donors on the post-transplant outcomes, such as 30-day and overall mortalities, was investigated. Graft survival was studied using the Kaplan-Meier survival analysis and log-rank test. Cox proportional hazards regression analyses, adjusted and unadjusted, were conducted to estimate the effect of CDC IR status on survival. The cumulative incidence functions were estimated, and the Gray tests were used for competing risk analyses on survival for the compared groups. For all analyses, $P$ values $<.05$ were considered statistically significant. All analyses were performed using SAS, version 9.4 (SAS Institute Inc, Cary, NC).

\section{RESULTS}

Of 24,751 adult primary heart transplant patients from 2004 to 2016 who met the study inclusion criteria, 3584 patients $(14.5 \%)$ received a heart from a CDC IR donor and 21,167 patients $(85.5 \%)$ received a heart from a non-CDC IR donor.

Prematching donors' and recipients' baseline characteristics are shown in Tables 1 and 2. The recipients of CDC IR grafts were slightly older, but not clinically significant, than recipients of non-CDC IR grafts (CDC IR vs non-CDC-IR; $53.5 \pm 12.4$ years old vs $52.8 \pm 12.6$ years old; $P=.003)$. There was a significantly greater percentage of male recipients (CDC IR vs non-CDC IR; $78.4 \%$ vs $74.6 \% ; P<.0001$ ) and male donors (CDC IR vs non-CDC IR; $77.9 \%$ vs $70.3 \% ; P<.0001)$ in the CDC IR cohort. The donors of CDC IR grafts were significantly younger (CDC IR vs non-CDC-IR; $29.8 \pm 9.4$ years old vs $32.1 \pm 12.0$ years old; $P<.0001)$. The incidences of diabetes and hypertension were significantly lower in the CDC IR grafts $(P=.03$ and $P<.0001$, respectively $)$, whereas the incidences of cocaine usage and hepatitis $C$ antibody positivity were significantly greater in the CDC

TABLE 1. A prematched analysis of recipients

\begin{tabular}{|c|c|c|c|c|}
\hline Recipients' baseline characteristics & Non-CDC IR $(n=21,167)$ & $\operatorname{CDC}$ IR $(n=3584)$ & $P$ value & ASMD \\
\hline Age, y & $52.8 \pm 12.6$ & $53.5 \pm 12.4$ & .003 & 0.053 \\
\hline Sex, male, n (\%) & $15,790(74.6)$ & 2809 (78.4) & $<.0001$ & 0.089 \\
\hline Body mass index, $\mathrm{kg} / \mathrm{m}^{2}$ & $27.1 \pm 4.9$ & $27.4 \pm 4.9$ & .0001 & 0.069 \\
\hline \multicolumn{5}{|l|}{ Medical history } \\
\hline Diabetes mellitus, n (\%) & $5717(27.1)$ & $984(27.5)$ & .59 & 0.01 \\
\hline On hemodialysis, $\mathrm{n}(\%)$ & $848(4.0)$ & $155(4.4)$ & .33 & 0.018 \\
\hline Etiology of heart failure & & & .25 & 0.113 \\
\hline Nonischemic cardiomyopathy, n (\%) & $10,281(48.6)$ & $1746(48.7)$ & & \\
\hline Ischemic cardiomyopathy, $\mathrm{n}(\%)$ & $7437(35.1)$ & $1221(34.1)$ & & \\
\hline Restrictive heart disease, $\mathrm{n}(\%)$ & $616(2.9)$ & $107(3.0)$ & & \\
\hline Congenital heart disease, $\mathrm{n}(\%)$ & $612(2.9)$ & $108(3.0)$ & & \\
\hline Hypertrophic cardiomyopathy, n (\%) & $482(2.3)$ & $79(2.2)$ & & \\
\hline Valvular heart disease, n (\%) & $379(1.8)$ & $53(1.5)$ & & \\
\hline Others, n (\%) & $1360(6.3)$ & $270(7.4)$ & & \\
\hline Total waitlist time, y & $0.58 \pm 0.97$ & $0.63 \pm 1.06$ & .009 & 0.049 \\
\hline Previous cardiac surgery, n (\%) & $10,362(49.2)$ & $1874(52.5)$ & .0002 & 0.067 \\
\hline \multicolumn{5}{|l|}{ Preoperative life support, n (\%) } \\
\hline Hospitalization in ICU, $\mathrm{n}(\%)$ & 6059 (28.7) & 1014 (28.6) & .43 & 0.028 \\
\hline IABP, n (\%) & $1162(5.5)$ & $247(6.9)$ & .0008 & 0.058 \\
\hline ECMO, n $(\%)$ & $118(0.6)$ & $27(0.8)$ & .16 & 0.024 \\
\hline Durable VAD, n (\%) & $7883(37.3)$ & $1533(43.2)$ & $<.0001$ & 0.12 \\
\hline Blood type & & & .22 & 0.056 \\
\hline $\mathrm{A}, \mathrm{n}(\%)$ & $8642(40.8)$ & $1422(39.7)$ & & \\
\hline $\mathrm{B}, \mathrm{n}(\%)$ & $3122(14.8)$ & $524(14.6)$ & & \\
\hline $\mathrm{AB}, \mathrm{n}(\%)$ & $1186(5.6)$ & $185(5.2)$ & & \\
\hline $\mathrm{O}, \mathrm{n}(\%)$ & $8217(38.8)$ & $1453(40.5)$ & & \\
\hline \multicolumn{5}{|l|}{ Preoperative data } \\
\hline Creatinine, $\mathrm{mg} / \mathrm{dL}$ & $1.33 \pm 0.82$ & $1.35 \pm 0.92$ & .16 & 0.027 \\
\hline Total bilirubin, $\mathrm{mg} / \mathrm{dL}$ & $1.11 \pm 1.83$ & $1.01 \pm 1.29$ & $<.0001$ & 0.064 \\
\hline
\end{tabular}

Categorical variables are expressed as n (\%), and continuous variables are expressed as mean \pm standard deviation. $C D C$, Centers for Disease Control and Prevention; $I R$, increased risk; $A S M D$, absolute standardized mean difference; $I C U$, intensive care unit; $I A B P$, intra-aortic balloon pump; $E C M O$, extracorporeal membrane oxygenation; $V A D$, ventricular assist device. 
TABLE 2. A prematched analysis of donors

\begin{tabular}{|c|c|c|c|c|}
\hline Donors' characteristics & Non-CDC IR $(n=21,167)$ & CDC IR $(n=3584)$ & $P$ value & ASMD \\
\hline Age, y & $32.1 \pm 12.0$ & $29.8 \pm 9.4$ & $<.0001$ & 0.213 \\
\hline Sex, male, n (\%) & $14,875(70.3)$ & $2791(77.9)$ & $<.0001$ & 0.174 \\
\hline Body mass index, $\mathrm{kg} / \mathrm{m}^{2}$ & $27.2 \pm 5.8$ & $26.6 \pm 5.3$ & $<.0001$ & 0.119 \\
\hline Left ventricular ejection fraction (\%) & $61.7 \pm 7.1$ & $61.1 \pm 6.9$ & $<.0001$ & 0.084 \\
\hline Allograft ischemic time, $\mathrm{h}$ & $3.2 \pm 1.1$ & $3.2 \pm 1.0$ & .202 & 0.023 \\
\hline \multicolumn{5}{|l|}{ Medical history } \\
\hline Diabetes mellitus, n (\%) & $703(3.3)$ & $92(2.6)$ & .03 & 0.04 \\
\hline Hypertension, n (\%) & $3158(14.5)$ & $395(11.2)$ & $<.0001$ & 0.111 \\
\hline Cocaine use, $\mathrm{n}(\%)$ & $2697(12.9)$ & $1213(35.5)$ & $<.0001$ & 0.548 \\
\hline Hepatitis C positive, $\mathrm{n}(\%)$ & $19(0.1)$ & $23(0.6)$ & $<.0001$ & 0.092 \\
\hline Coronary artery disease, $\mathrm{n}(\%)$ & $499(2.4)$ & $70(1.9)$ & $<.0001$ & 0.099 \\
\hline Blood type & & & .07 & 0.022 \\
\hline $\mathrm{A}, \mathrm{n}(\%)$ & $7603(36.1)$ & 1239 (34.6) & & \\
\hline $\mathrm{B}, \mathrm{n}(\%)$ & 2360 (11.2) & 379 (10.6) & & \\
\hline $\mathrm{AB}, \mathrm{n}(\%)$ & $457(2.2)$ & 68 (1.9) & & \\
\hline $\mathrm{O}, \mathrm{n}(\%)$ & $10,720(50.6)$ & $1898(53.0)$ & & \\
\hline
\end{tabular}

Categorical variables are expressed as n (\%), and continuous variables are expressed as mean \pm standard deviation. $C D C$, Centers for Disease Control and Prevention; $I R$, increased risk; $A S M D$, absolute standardized mean difference.

IR grafts $(P<.0001$ for both comparisons). The mean body mass index of recipients was $27.4 \pm 4.9 \mathrm{~kg} / \mathrm{m}^{2}$ in the CDC IR group and $27.1 \pm 4.9 \mathrm{~kg} / \mathrm{m}^{2}$ in the non-CDC IR group $(P=.0001)$. The percentages of blood type $\mathrm{O}$ recipients (CDC IR vs non-CDC IR; $40.5 \%$ vs $38.8 \% ; P=.22$ ) and donors (CDC IR vs non-CDC IR; $53.0 \%$ vs $50.6 \%$; $P=.07)$ were greater in the CDC IR grafts, but no blood type differences were observed in recipients or donors receiving CDC IR versus non-CDC IR grafts. The total waiting period was significantly longer in the CDC IR group than in the non-CDC IR group (CDC IR vs non-CDC-IR; $0.63 \pm 1.06$ years vs $0.58 \pm 0.97$ years; $P=.009$ ). Greater incidence of blood type $\mathrm{O}$ recipients within the cohort of CDC IR graft recipients was likely to be translated into a significant difference in the total waitlist time between the cohorts.

"Short-term mechanical circulatory assist" usage, such as IABP and ECMO, before transplant was significantly greater in CDC IR recipients, with 247 (6.9\%) patients requiring an IABP and $27(0.8 \%)$ patients requiring ECMO compared with non-CDC IR recipients, where $1162(5.5 \%)$ patients used an IABP and $118(0.6 \%)$ patients were on ECMO (IABP and ECMO; $P=.0008$ and .16). "Long-term mechanical circulatory assist" usage, such as durable ventricular assist device, occurred significantly more frequently in CDC IR recipients than in non-CDC IR recipients (CDC IR vs non-CDC IR; $43.2 \%$ vs $37.3 \% ; P<.0001)$. The rate of hospital admissions in the intensive care unit before transplant was not significantly different between CDC IR recipients $(28.6 \%)$ and non-CDC IR recipients $(28.7 \%, P=.43)$. The aforementioned results, greater incidence of mechanical circulatory support usage, suggest that the cohort of CDC IR patients included relatively sicker recipients.

Of those 24,751 recipients, 6304 transplants were successfully matched (CDC IR, $\mathrm{n}=3152$; non-CDC IR, $\mathrm{n}=3152$ ) using the matching algorithm. In the matched cohort, mean age for adult primary heart transplants was 53.3 years old. In total, 4908 recipients $(77.9 \%)$ were men, 3120 recipients $(49.5 \%)$ had nonischemic cardiomyopathy, and 2145 recipients $(34.0 \%)$ had ischemic cardiomyopathy. There were no significant differences in recipient or donor baseline characteristics between CDC IR and non-CDC IR groups after matching (Tables 3 and 4).

Among the post-transplant outcomes, acute myocardial rejection episodes, defined as necessitating treatment with antirejection medications at least once, were found to be more often in the CDC-IR group (CDC IR, $\mathrm{n}=358$ [11.4\%]; non-CDC IR, $\mathrm{n}=304$ [9.6\%]; $P=.03$ ), whereas post-transplant pacemaker placements were less frequent in the CDC IR group (CDC IR, $\mathrm{n}=80$ [2.6\%]; non-CDC IR, $\mathrm{n}=111[3.5 \%] P=.02)$. To assess the effect of IR status on survival, time-to-event survival analyses were conducted on the matched cohort. The $P$ values of the log-rank tests on the Kaplan-Meier survival estimations of 2 matched groups were 0.84 for 30 -day mortality and 0.57 for overall survival (Figures 1 and 2). Unadjusted and adjusted hazard ratios for 30-day mortality and overall survival were not statistically significant (Table 5). In particular, hazard ratios of overall survival were 0.97 for the unadjusted analysis and 0.94 for the adjusted analysis $(P=.84$ and .62 , respectively). Interestingly, there was no significant difference in the cumulative incidence of coronary vasculopathy $(P=.39$, Gray test) or re-heart transplant $(P=.11$, Gray test) during follow-up. However, there was a significant difference in 
TABLE 3. A propensity score-matched analysis of recipients

\begin{tabular}{|c|c|c|c|c|}
\hline Recipients' baseline characteristics & Non-CDC IR $(n=3152)$ & $\operatorname{CDC}$ IR $(n=3152)$ & $P$ value & ASMD \\
\hline Age, $y$ & $53.3 \pm 12.4$ & $53.4 \pm 12.3$ & .87 & 0.004 \\
\hline Sex, male, n (\%) & $2448(77.7)$ & $2460(78.1)$ & .72 & 0.006 \\
\hline Body mass index, $\mathrm{kg} / \mathrm{m}^{2}$ & $27.5 \pm 4.9$ & $27.4 \pm 4.9$ & .44 & 0.0196 \\
\hline \multicolumn{5}{|l|}{ Medical history } \\
\hline Diabetes mellitus, n (\%) & $863(27.4)$ & 870 (27.6) & .84 & 0.0176 \\
\hline On hemodialysis, n (\%) & $119(3.8)$ & $135(4.3)$ & .31 & 0.005 \\
\hline Etiology of heart failure & & & .78 & 0.0392 \\
\hline Nonischemic cardiomyopathy, n (\%) & $1578(50.1)$ & $1542(48.9)$ & & \\
\hline Ischemic cardiomyopathy, $\mathrm{n}(\%)$ & $1065(33.8)$ & $1080(34.3)$ & & \\
\hline Restrictive heart disease, $\mathrm{n}(\%)$ & $102(3.2)$ & $95(3.0)$ & & \\
\hline Congenital heart disease, $\mathrm{n}(\%)$ & $81(2.6)$ & $91(2.9)$ & & \\
\hline Hypertrophic cardiomyopathy, n (\%) & $65(2.1)$ & $68(2.2)$ & & \\
\hline Valvular heart disease, $\mathrm{n}(\%)$ & $35(1.1)$ & $47(1.5)$ & & \\
\hline Others, n (\%) & $226(7.2)$ & $229(7.3)$ & & \\
\hline Total waitlist time, $y$ & $0.6 \pm 0.92$ & $0.6 \pm 0.96$ & .84 & 0.005 \\
\hline Previous cardiac surgery, $\mathrm{n}(\%)$ & $1641(52.1)$ & $1653(52.4)$ & .76 & 0.0258 \\
\hline \multicolumn{5}{|l|}{ Preoperative life support, $\mathrm{n}(\%)$} \\
\hline Hospitalization in ICU, n (\%) & $534(16.9)$ & $525(16.7)$ & .87 & 0.0231 \\
\hline IABP, n (\%) & $207(6.6)$ & $224(7.1)$ & .4 & 0.0214 \\
\hline ECMO, n (\%) & $13(0.4)$ & $22(0.7)$ & .13 & 0.0384 \\
\hline Durable VAD, n (\%) & $1389(44.1)$ & $1357(43.1)$ & .42 & 0.0205 \\
\hline Blood Type & & & .37 & 0.0314 \\
\hline $\mathrm{A}, \mathrm{n}(\%)$ & $1274(40.4)$ & $1276(40.5)$ & & \\
\hline $\mathrm{B}, \mathrm{n}(\%)$ & $410(13.0)$ & $452(14.3)$ & & \\
\hline $\mathrm{AB}, \mathrm{n}(\%)$ & $191(6.1)$ & $174(5.5)$ & & \\
\hline $\mathrm{O}, \mathrm{n}(\%)$ & $1277(40.5)$ & $1250(39.7)$ & & \\
\hline \multicolumn{5}{|l|}{ Preoperative data } \\
\hline Creatinine, $\mathrm{mg} / \mathrm{dL}$ & $1.34 \pm 0.84$ & $1.35 \pm 0.92$ & .79 & 0.0098 \\
\hline Total bilirubin, $\mathrm{mg} / \mathrm{dL}$ & $1.02 \pm 1.28$ & $1 \pm 1.28$ & .66 & 0.0111 \\
\hline
\end{tabular}

Categorical variables are expressed as n (\%), and continuous variables are expressed as mean \pm standard deviation. $C D C$, Centers for Disease Control and Prevention; $I R$, increased risk; $A S M D$, absolute standardized mean difference; $I C U$, intensive care unit; $I A B P$, intra-aortic balloon pump; $E C M O$, extracorporeal membrane oxygenation; $V A D$, ventricular assist device.

the cumulative incidence of pacemaker placement $(P=.004$, Gray test).

\section{DISCUSSION}

In this study, we aimed to compare outcomes of adult primary OHT recipients receiving CDC IR versus non-CDC IR donor grafts using the UNOS database. The most important finding of this study is that patients receiving IR donor grafts were not subjected to an increased risk of death during either the short-term or overall follow-up after transplant compared with those using non-IR donor grafts.

Historically, many treatment options have been developed for patients with end-stage heart failure, among which OHT remains the gold standard. ${ }^{2}$ However, approximately $10 \%$ of all candidates on the waiting list for solid-organ transplantation die each year without receiving an organ. To surmount the organ shortage challenge, we have previously proposed alternative approaches to maximize organ allocation by using marginally acceptable organs, ${ }^{3}$ harvesting donor hearts from distant locations, accepting longer cold ischemic times, and applying a domino heart transplantation as a uniquely efficacious surgical strategy. ${ }^{4}$ According to the $\mathrm{CDC}$, drug overdose was the leading cause of injury death, more so than car accidents and homicide, in 2013. ${ }^{8}$ Together with the national epidemic of drug abuse and overdose, the percentage of donors with a history of injection drug use (ie, IR donor) has been increasing. Moreover, a significant number of medically suitable organs from IR donors are not transplanted. ${ }^{5}$ Therefore, one possible solution could be to maximize the use of IR donors, given the number of high-risk donors are anticipated to increase in the future.

In our prematched analysis, we showed that recipients receiving CDC IR grafts were older, had a greater body mass index, longer total waiting period, and greater percentage of blood type $\mathrm{O}$ than those receiving non-CDC IR grafts. In addition, the cohort receiving CDC IR grafts showed a greater incidence of short-term and long-term 
TABLE 4. A propensity score-matched analysis of donors

\begin{tabular}{|c|c|c|c|c|}
\hline Donors' characteristics & Non-CDC IR $(n=3152)$ & $\operatorname{CDC}$ IR $(n=3152)$ & $P$ value & ASMD \\
\hline Age, y & $29.5 \pm 10.6$ & $29.6 \pm 9.3$ & .65 & 0.012 \\
\hline Sex, male, $\mathrm{n}(\%)$ & $2467(78.3)$ & $2444(77.5)$ & .49 & 0.018 \\
\hline Body mass index, $\mathrm{kg} / \mathrm{m}^{2}$ & $26.5 \pm 5.2$ & $26.6 \pm 5.3$ & .56 & 0.015 \\
\hline Left ventricular ejection fraction ( $\%)$ & $61.0 \pm 7.0$ & $61.2 \pm 6.9$ & .47 & 0.015 \\
\hline Allograft ischemic time, $h$ & $3.2 \pm 1.0$ & $3.2 \pm 1.0$ & .25 & 0.029 \\
\hline \multicolumn{5}{|l|}{ Medical history } \\
\hline Diabetes mellitus, n (\%) & $76(2.4)$ & $82(2.6)$ & .63 & 0.018 \\
\hline Hypertension, $\mathrm{n}(\%)$ & $361(11.5)$ & $352(11.2)$ & .72 & 0.009 \\
\hline Cocaine usage, $\mathrm{n}(\%)$ & $1080(34.3)$ & $1089(34.6)$ & .81 & 0.006 \\
\hline Hepatitis $\mathrm{C}$ positive, $\mathrm{n}(\%)$ & $5(0.2)$ & $6(0.2)$ & 1 & 0.008 \\
\hline Coronary artery disease, $\mathrm{n}(\%)$ & $58(1.8)$ & $63(2.0)$ & .67 & 0.024 \\
\hline Blood type & & & .42 & 0.035 \\
\hline $\mathrm{A}, \mathrm{n}(\%)$ & $1120(35.5)$ & $1116(35.4)$ & & \\
\hline $\mathrm{B}, \mathrm{n}(\%)$ & $300(9.5)$ & 335 (10.6) & & \\
\hline $\mathrm{AB}, \mathrm{n}(\%)$ & $73(2.3)$ & $63(2.0)$ & & \\
\hline $\mathrm{O}, \mathrm{n}(\%)$ & 1659 (52.6) & $1638(52.0)$ & & \\
\hline
\end{tabular}

Categorical variables are expressed as n (\%), and continuous variables are expressed as mean \pm standard deviation. $C D C$, Centers for Disease Control and Prevention; $I R$, increased risk; $A S M D$, absolute standardized mean difference.

mechanical circulatory support usage, suggesting that recipients receiving CDC IR grafts might be relatively sicker than those receiving non-CDC IR grafts. This can likely be explained by the eagerness and urgency for organ acceptance due to the severity of clinical status in this recipient population, despite potential concerns for CDC IR donor grafts.

In this study, propensity matched cohorts were generated to mitigate any potential confounding factors.
After propensity matching, our data demonstrated that 30-day and overall mortality at follow-up was not impacted by the CDC IR status. Importantly, this study revealed that CDC IR graft use appeared to have some impact on acute rejection episodes after OHT, which was not found in previous reports, including 1 previous UNOS study. However, this finding did not adversely affect adjusted overall outcomes, which is consistent with previous reports. ${ }^{9-11}$

Matched 30-day Survival Kaplan-Meier Estimates - IR Donors vs Non-IR Donors With Number of Subjects at Risk and 95\% Confidence Limits

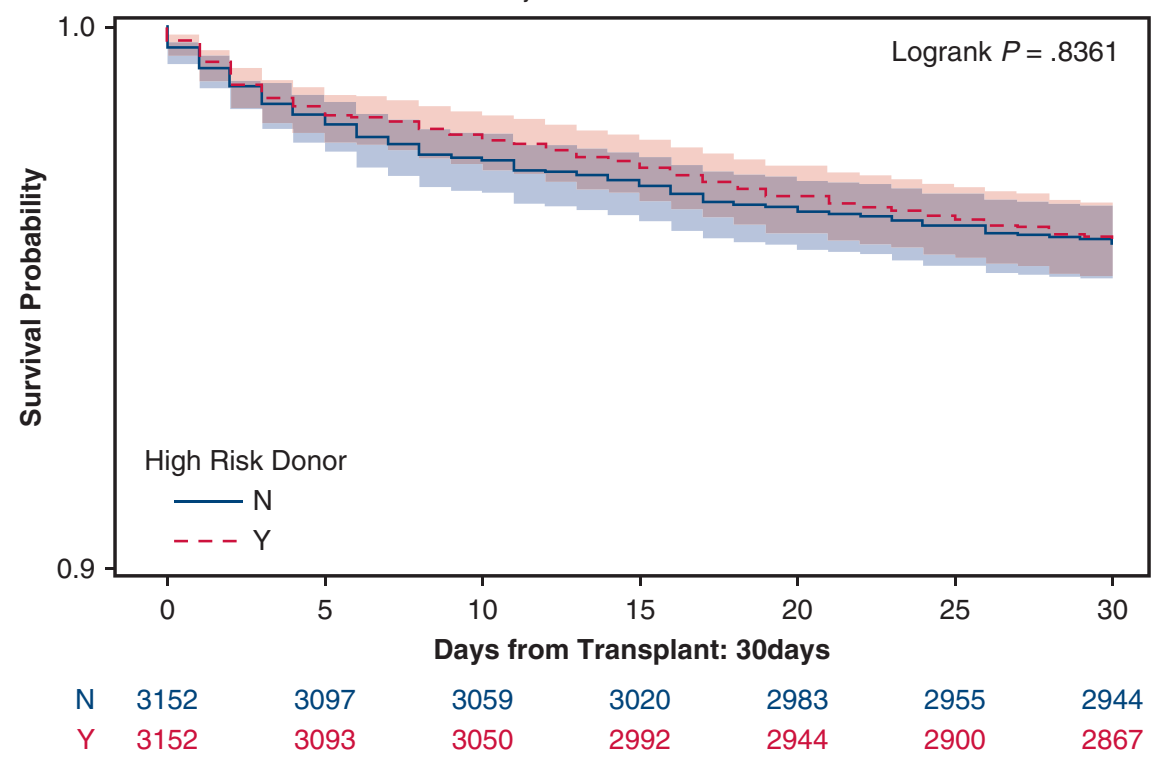

FIGURE 1. Thirty-day survival Kaplan-Meier estimates comparing propensity matched patients who received increased risk (red line) versus nonincreased risk donor hearts (blue line $)(P=.84$, log-rank test). $I R$, Increased risk. 


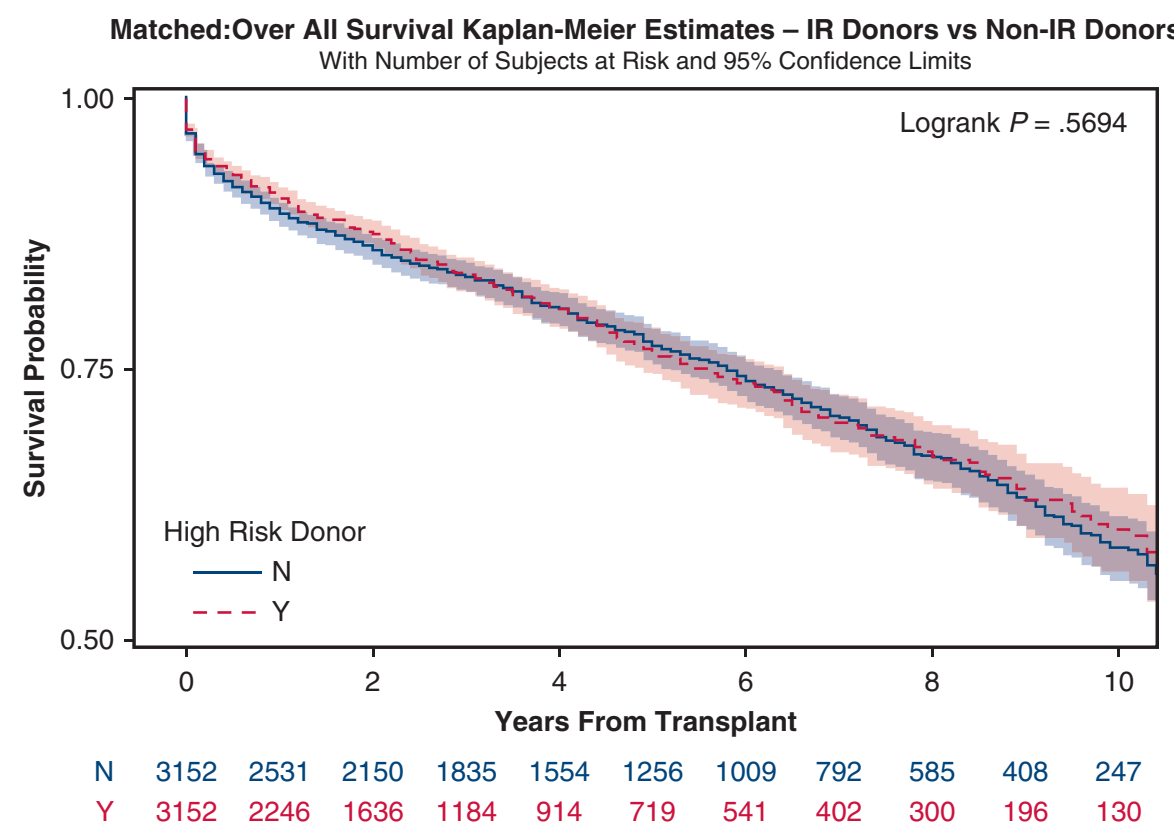

FIGURE 2. Overall survival Kaplan-Meier estimates comparing propensity matched patients who received increased risk (red line) versus nonincreased risk donor hearts (blue line) $(P=.57, \log$-rank test). $I R$, Increased risk.

Interestingly, post-transplant permanent pacemaker implantation was less frequent in the cohort receiving CDC IR grafts. The need for permanent pacemaker implantation after OHT still remains a significant issue despite a general shift from the biatrial surgical implantation technique toward the bicaval technique. ${ }^{12}$ In addition, short-term use of positive chronotropic drugs in the management of postoperative bradycardia has been implemented to address this challenge. The indication for pacing in patients after OHT is complex, but permanent pacemaker implantation could possibly be a marker for worse outcomes. ${ }^{13}$ Onset of conducting system disease after OHT may be associated with an overt or occult rejection process, including cardiac allograft vasculopathy. Thus, we speculate that CDC IR grafts may potentially represent greater quality grafts than non-CDC IR grafts.

TABLE 5. Survival effects of CDC-IR donor hearts in Cox models on the matched compare groups

\begin{tabular}{ccccc}
\hline Models & \multicolumn{5}{c}{ Outcomes } & $\begin{array}{c}\text { Hazard ratio } \\
\text { (IR vs Non-IR) }\end{array}$ & $\mathbf{9 5 \%} \mathbf{C I}$ & $\boldsymbol{P}$ value \\
\hline Unadjusted & & & & \\
& 30-d mortality & 0.97 & $0.87-1.08$ & .57 \\
& Overall mortality & 0.97 & $0.76-1.25$ & .84 \\
\hline Adjusted $^{*}$ & & & & \\
& 30-d mortality & 0.97 & $0.87-1.08$ & .57 \\
& Overall mortality & 0.94 & $0.73-1.21$ & .62 \\
\hline
\end{tabular}

$I R$, Increased risk; $C I$, confidence interval. *Results adjusted in Cox proportional hazards model by baseline characteristics—age, sex, body mass index, medical history (diabetes, renal failure, liver function), etiology of heart failure, total waitlist time, previous cardiac surgery, preoperative life support (intra-aortic balloon pump, extracorporeal membrane oxygenation, durable ventricular assist device), blood type.
Although previous studies, ${ }^{9-11}$ including our current one, showed that both recipients of CDC IR and non-CDC IR grafts had similar overall survival probability, one of the potential principal factors leading to the underuse of high-risk donors was the concern of transmitting infections, such as hepatitis $\mathrm{B}$ virus, hepatitis $\mathrm{C}$ virus (HCV), and HIV. This concern was formulated decades ago, but with the development of more sensitive and rapid screening tests, such as nucleic acid testing (NAT), the risk of infection transmission has substantially decreased. ${ }^{14}$ Although NAT data were not available in the UNOS database, the advent of routine NAT testing in the last several years has shortened the period for detection of $\mathrm{HCV}$ and HIV. Another interesting aspect that may warrant further investigation would be the potential rates of seroconversion as well as morbidity associated with these transmitting infections such as HCV and HIV. However, only 24 heart recipients either had $\mathrm{HCV}$ or received an organ from an HCV-positive donor between 2004 and 2005 in our study, not sufficient to draw a meaningful conclusion. ${ }^{15}$

Related to this, the advent of curative and well-tolerated medications for infections such as HCV will reduce the infectious risks. Furthermore, Gaffey and colleagues ${ }^{9}$ reported that the risk of transmission of infection from IR donors was minimal. These findings will hopefully inspire transplant surgeons and patients to be more willing to accept CDC IR grafts, therefore reducing disposal of these donor grafts and increasing the donor pool, which may eventually shorten the waiting period for organs and ultimately benefit all recipients.

This study has several limitations. Given the retrospective nature of this study, specific data, such as hepatitis B 
virus, $\mathrm{HCV}$, and $\mathrm{HIV}$ status of transplant recipients, panel reactive antibody, and crossmatch results, were not available, as they were not routinely collected by UNOS. Similarly, we were unable to determine acute rejection episodes in a more granular fashion, such as timing, frequency, and associated heart failure episodes and hospitalization. Further follow-up information as to the rate of rejection episodes outside of the initial hospital visit may also be important to investigate in future studies. More detailed information regarding permanent pacemaker implantation, such as pathogenesis, natural history, indication, and optimal management, was also limited. The quality of life of the transplant recipients between the 2 groups was not evaluated, given the nature of the study. Looking forward, the effect of transplant technique (eg, biatrial vs bicaval) and allograft ischemic time may be of interest for further study. It may also be greatly beneficial to examine geographic patterns by evaluating the rates of donor heart usage and the graft outcomes in different UNOS regions.

\section{CONCLUSIONS}

The equivalent recipient survival reported at long-term follow-up is encouraging with respect to the use of CDC IR donor grafts. Increased use of CDC IR donor grafts may potentially improve the persistent and worsening shortage of available donor organs and shorten the waitlist time for heart transplantation.

\section{Conflict of Interest Statement}

Authors have nothing to disclose with regard to commercial support.

The data reported here have been supplied by the United Network for Organ Sharing (UNOS) as the contractor for the Organ Procurement and Transplantation Network. UNOS is the private, nonprofit organization that manages the nation's organ transplant system under contract with the federal government. This system serves as the model for transplant systems around the world.

\section{References}

1. Hunt SA, Abraham WT, Chin MH, Feldman AM, Francis GS, Ganiats TG, et al ACC/AHA 2005 guideline update for the diagnosis and management of chronic heart failure in the adult: a report of the American College of Cardiology/American Heart Association Task Force on Practice Guidelines (Writing Committee to update the 2001 guidelines for the evaluation and management of heart failure). Circulation. 2005;112:e154-235.

2. Joyce DL, Li Z, Edwards LB, Kobashigawa JA, Daly RC. Predicting 1-year cardiac transplantation survival using a donor-recipient risk-assessment tool. $J$ Thorac Cardiovasc Surg. 2018:155:1580-90.

3. Okamura H, Woo YJ. Ex vivo allograft mitral valve leaflet repair prior to orthotopic heart transplantation. J Card Surg. 2014;29:424-6.

4. Shudo Y, Ma M, Boyd JH, Woo YJ. Current status of domino heart transplantation. J Card Surg. 2017:32:229-32.

5. U.S. Department of Health and Human Services. Organ Procurement and Transplantation Network. Available at: https://optn.transplant.hrsa.gov. Accessed November 7, 2018

6. Austin PC. Optimal caliper widths for propensity-score matching when estimating differences in means and differences in proportions in observational studies. Pham Stat. 2011;10:150-61.

7. Goldstone AB, Chiu P, Baiocchi M, Lingala B, Patrick WL, Fischbein MP, et al. Mechanical or biologic prostheses for aortic-valve and mitral-valve replacement. N Engl J Med. 2017;377:1847-57.

8. Woolley AE, Baden LR. Increasing access to thoracic organs from donors infected with hepatitis $\mathrm{C}$ : a previous challenge-now an opportunity. J Heart Lung Transplant. 2018;37:681-3.

9. Gaffey AC, Doll SL, Thomasson AM, Venkataraman C, Chen CW, Goldberg LR, et al. Transplantation of "high-risk" donor hearts: implications for infection. $J$ Thorac Cardiovasc Surg. 2016;152:213-20.

10. Sahulee R, Lytrivi ID, Savla JJ, Rossano JW. Centers for disease control "highrisk" donor status does not significantly affect recipient outcome after heart transplantation in children. J Heart Lung Transplant. 2014;33:1173-7.

11. Gaffey AC, Cucchiara AJ, Goldberg LR, Blumberg EA, Acker MA, Atluri P. Transplantation of center for disease control "high-risk" donor hearts does not adversely impact long-term outcomes in adults. J Card Fail. 2016;22:376-82.

12. Shudo Y, Wang H, Woo YJ. A modified technique for orthotopic heart transplantation to minimize warm ischaemic time. Eur J Cardiothorac Surg. 2018;53: 1089-90.

13. Jones DG, Mortsell DH, Rajaruthnam D, Hamour I, Hussain W, Markides V, et al. Permanent pacemaker implantation early and late after heart transplantation: clinical indication, risk factors and prognostic implications. J Heart Lung Transplant. 2011;30:1257-65.

14. Fischer SA, Avery RK. Screening of donor and recipient prior to solid organ transplantation. Am J Transplant. 2009:9:S7-18.

15. Levitsky J, Formica RN, Bloom RD, Charlton M, Curry M, Friedewald J, et al. The American Society of Transplantation consensus conference on the use of hepatitis C viremic donors in solid organ transplantation. Am J Transplant. 2017;17:2790-802.

Key Words: orthotopic heart transplantation, propensitymatched analysis, increased-risk donor, UNOS, CDC 